\title{
Erratum to: The 5As team patient study: patient perspectives on the role of primary care in obesity management
}

\author{
Jacqueline Torti ${ }^{1,2 \dagger}$, Thea Luig ${ }^{1,3 \dagger}$, Michelle Borowitz ${ }^{4}$, Jeffrey A. Johnson ${ }^{2,5}$, Arya M. Sharma ${ }^{3,5}$ \\ and Denise L. Campbell-Scherer ${ }^{1,5^{*}}$
}

\section{Erratum}

After publication of the original article [1], it came to the authors' attention that the affiliations were incorrect.

Affiliation 1 (Department of Family Medicine, University of Alberta) incorrectly contained 'Clinical Research Unit', and the postcode was incorrect. Affiliation 5 (Alberta Diabetes Institute, University of Alberta) was mistakenly included as 'Department of Medicine, Obesity Research \& Management, University of Alberta'.

In addition, in the list of authors, Jeffrey A. Johnson should be affiliated to institutions 2 and 5, and Arya. A. Sharma should have been affiliation to institutions 3 and 5 .

The list of authors and affiliations is correct in this erratum. The 'Author details' section of the article should have read as follows:

\section{Author details}

${ }^{1}$ Department of Family Medicine, University of Alberta, Edmonton, AB, Canada T6G 2T4. ${ }^{2}$ School of Public Health, University of Alberta, Edmonton, AB, Canada T6G 2E3. ${ }^{3}$ Department of Medicine, University of Alberta, Edmonton, AB, Canada T6G 2E1. ${ }^{4}$ Department of Anthropology, University of Alberta, Edmonton, AB, CanadaT6G 2H4. ${ }^{5}$ Alberta Diabetes Institute, University of Alberta, Edmonton, AB, Canada T6G 2T9.

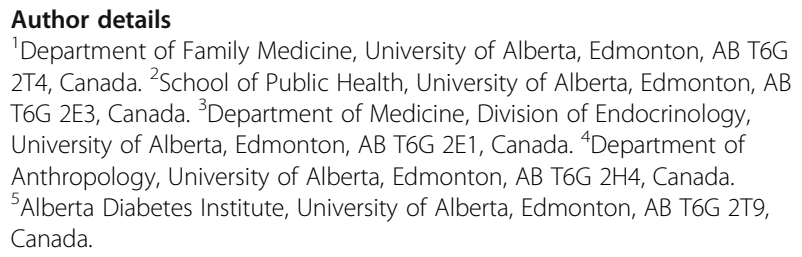

Published online: 04 May 2017

\author{
Reference \\ 1. Torti J, Luig T, Borowitz M, Johnson JA, Sharma AM, Campbell-Scherer DL. The \\ 5 As team patient study: patient perspectives on the role of primary care in \\ obesity management. BMC Fam Pract. 2017;18:19. doi:10.1186/s12875-017-0596-2.
}

\footnotetext{
* Correspondence: denise.campbell-scherer@ualberta.ca

${ }^{\dagger}$ Equal contributors

'Department of Family Medicine, University of Alberta, Edmonton, AB T6G

2T4, Canada

${ }^{5}$ Alberta Diabetes Institute, University of Alberta, Edmonton, AB T6G 2T9,

Canada
} 